\title{
POINCARÉ-LELONG APPROACH TO UNIVERSALITY AND SCALING OF CORRELATIONS BETWEEN ZEROS
}

\author{
PAVEL BLEHER, BERNARD SHIFFMAN, AND STEVE ZELDITCH
}

\begin{abstract}
This note is concerned with the scaling limit as $N \rightarrow \infty$ of $n$-point correlations between zeros of random holomorphic polynomials of degree $N$ in $m$ variables. More generally we study correlations between zeros of holomorphic sections of powers $L^{N}$ of any positive holomorphic line bundle $L$ over a compact Kähler manifold. Distances are rescaled so that the average density of zeros is independent of $N$. Our main result is that the scaling limits of the correlation functions and, more generally, of the "correlation forms" are universal, i.e. independent of the bundle $L$, manifold $M$ or point on $M$.
\end{abstract}

\section{INTRODUCTION}

This note is a companion to our article [BSZ], in which we study the correlations between the zeros of a random holomorphic section $s \in H^{0}\left(M, L^{N}\right)$ of a power $L^{N}$ of a positive line bundle $L \rightarrow M$ over a compact $m$-dimensional complex manifold $M$. Since the hypersurface volume of the zeros of a section of $L^{N}$ in a ball $U$ around a given point $z_{0}$ is $\sim N \mathrm{Vol}(U)$, we rescale $U \rightarrow \sqrt{N} U$ to get a density of zeros independent of $N$. After expanding $U$ this way, all manifolds and line bundles appear asymptotically alike, and it is natural to ask if the local statistics of zeros are universal, i.e. independent of $L, M, \omega$ and $z_{0}$. To define our statistics, we first provide $H^{0}\left(M, L^{N}\right)$ with a natural Gaussian measure (see $\S \S 1.1$ (1.2). The local statistics are measured by the scaled $n$-point zero correlation forms $\vec{K}_{n}^{N}\left(\frac{z^{1}}{\sqrt{N}}, \ldots, \frac{z^{n}}{\sqrt{N}}\right), z^{j} \in \sqrt{N} U$ (see $\oint[1.3)$. They are smooth forms on the "off-diagonal" domain $\mathcal{G}_{n}^{m} \subset C^{m n}$ consisting of $n$-tuples of distinct points $z^{j} \in \mathbb{C}^{m}$, and their norms define scaled zero correlation measures $\widetilde{K}_{n}^{N}\left(\frac{z^{1}}{\sqrt{N}}, \ldots, \frac{z^{n}}{\sqrt{N}}\right)$. (The correlation forms extend to all of $\mathbb{C}^{m n}$ as currents of order 0 , and hence the same holds for the correlation measures.) In [BSZ, we used geometric probability methods and a (universal) scaled Szegö kernel to prove that there exist universal limits as $N \rightarrow \infty$ of these correlation measures and more generally of the correlations between simultaneous zeros of $k \leq m$ sections. Here we use a complex analytic approach based on the Poincaré-Lelong formula for the currents of integration over the zero set of a section, together with the scaled Szegö kernel from [BSZ], to give a proof of universality for the correlation forms. This approach, although limited to the hypersurface case, allows for a result on the level of forms and a somewhat simpler proof.

Our universality theorem is as follows:

Date: March 4, 1999.

Research partially supported by NSF grants \#DMS-9623214 (first author), \#DMS-9800479 (second author), \#DMS-9703775 (third author). 
MAIN TheOREM. There is a universal current $\vec{K}_{n}^{\infty} \in \mathcal{D}^{\prime(m-1) n,(m-1) n}\left(\mathbb{C}^{m n}\right)$ such that the following holds: suppose that $(L, h)$ is a positive Hermitian line bundle on an $m$-dimensional compact complex manifold $M$, and let $\vec{K}_{n}^{N}$ be the n-point zero correlation current on $M^{n}$. Suppose $z^{0} \in M$ and choose local holomorphic coordinates in $M$ about $z^{0}$ such that $\left.\Theta_{h}\right|_{z^{0}}=$ $\partial \bar{\partial}|z|^{2}$. Then

$$
\vec{K}_{n}^{N}\left(\frac{z^{1}}{\sqrt{N}}, \ldots, \frac{z^{n}}{\sqrt{N}}\right)=\vec{K}_{n}^{\infty}\left(z^{1}, \ldots, z^{n}\right)+O\left(\frac{1}{\sqrt{N}}\right) .
$$

Furthermore, $\vec{K}_{n}^{\infty}$ is a smooth form on the off-diagonal domain $\mathcal{G}_{n}^{m}$, and the error term has $k^{\text {th }}$ order derivatives $\leq \frac{C_{A, k}}{\sqrt{N}}$ on each compact subset $A \subset \mathcal{G}_{n}^{m}, \forall k \geq 0$.

Our method leads to integral formulae for these universal limit forms, although the details rapidly become complicated as the number $n$ of points increase. For the case $m=2$, we carry out the calculation in complete detail in dimension one and also use the method to obtain an explicit formula for the scaling limit pair correlation measures in all dimensions (Theorems 4.1 and 4.2). In particular, our formula gives the scaling limit pair correlations for $\mathrm{SU}(m+1)$-polynomials (which are sections of powers of the hyperplane bundle over complex projective space $\left.\mathbb{C P}^{m}\right)$. The universal formula in dimension one agrees, as it must, with that of Bogomolny-Bohigas-Leboeuf [BBD and Hannay $\mathrm{Ba}$ in the case of random $\mathrm{SU}(2)$-polynomials. Similar formulas for correlations of zeros of real polynomials were given in $\mathrm{BD}$.

Before we get started on the proof, a few heuristic remarks on correlation measures and forms may be helpful. Roughly speaking, $\widetilde{K}_{n}^{N}\left(z^{1}, \ldots, z^{n}\right)$ gives the conditional probability density of the zero divisor of a random section $s$ (simultaneously) intersecting small balls around $z^{k+1}, \ldots, z^{n}$, given that the zero divisor (simultaneously) intersects small balls around $z^{1}, \ldots, z^{k}$. The correlation form $\vec{K}_{n}^{N}$ gives a more refined conditional probability: Let $Y$ denote the set of holomorphic tangent hyperplanes in $M$. (We can identify $Y$ with the projectivized holomorphic cotangent bundle of $M$.) Then $\vec{K}_{n}^{N}$ gives the conditional probability that the zero divisor has tangent hyperplanes in small balls in $Y$ above $z^{k+1}, \ldots, z^{n}$, given that it has tangents in small balls above $z^{1}, \ldots, z^{k}$.

Acknowledgment. The first draft of this paper was completed while the third author was visiting the Erwin Schrodinger Institute in July 1998. He wishes to thank that institution for its hospitality and financial support.

\section{Notation}

We summarize here the notation from complex analysis that we will need in the proof. This notation is the same as in [SZ] and [BSZ, except that different normalizations for the metric and volume form are used in $\mathrm{SZ}$.

1.1. Complex geometry. We denote by $(L, h) \rightarrow M$ a holomorphic line bundle with smooth Hermitian metric $h$ whose curvature form

$$
\Theta_{h}=-\partial \bar{\partial} \log \left\|e_{L}\right\|_{h}^{2},
$$

is a positive $(1,1)$-form. Here, $e_{L}$ is a local non-vanishing holomorphic section of $L$ over an open set $U \subset M$, and $\left\|e_{L}\right\|_{h}=h\left(e_{L}, e_{L}\right)^{1 / 2}$ is the $h$-norm of $e_{L}$. As in [BSZ], we give $M$ the 
Hermitian metric corresponding to the Kähler form $\omega=\frac{\sqrt{-1}}{2} \Theta_{h}$ and the induced Riemannian volume form

$$
d V_{M}=\frac{1}{m !} \omega^{m}
$$

We denote by $H^{0}\left(M, L^{N}\right)$ the space of holomorphic sections of $L^{N}=L \otimes \cdots \otimes L$. The metric $h$ induces Hermitian metrics $h^{N}$ on $L^{N}$ given by $\left\|s^{\otimes N}\right\|_{h^{N}}=\|s\|_{h}^{N}$. We give $H^{0}\left(M, L^{N}\right)$ the Hermitian inner product

$$
\left\langle s_{1}, s_{2}\right\rangle=\int_{M} h^{N}\left(s_{1}, s_{2}\right) d V_{M} \quad\left(s_{1}, s_{2} \in H^{0}\left(M, L^{N}\right)\right),
$$

and we write $|s|=\langle s, s\rangle^{1 / 2}$.

For a holomorphic section $s \in H^{0}\left(M, L^{N}\right)$, we let $Z_{s}$ denote the current of integration over the zero divisor of $s$ :

$$
\left(Z_{s}, \varphi\right)=\int_{Z_{s}} \varphi, \quad \varphi \in \mathcal{D}^{m-1, m-1}(M)
$$

The Poincaré-Lelong formula (see e.g., [GH]) expresses the integration current of a holomorphic section $s=g e_{L}^{\otimes N}$ in the form:

$$
Z_{s}=\frac{i}{\pi} \partial \bar{\partial} \log |g|=\frac{i}{\pi} \partial \bar{\partial} \log \|s\|_{h^{N}}+N \omega .
$$

We also denote by $\left|Z_{s}\right|$ the Riemannian $(2 m-2)$-volume along the regular points of $Z_{s}$, regarded as a measure on $M$ :

$$
\left(\left|Z_{s}\right|, \varphi\right)=\int_{Z_{s}^{\text {reg }}} \varphi d \mathrm{Vol}_{2 m-2}=\frac{1}{(m-1) !} \int_{Z_{s}^{\text {reg }}} \varphi \omega^{m-1}
$$

i.e., $\left|Z_{s}\right|$ is the total variation measure of the current of integration over $Z_{s}$ :

$$
\left|Z_{s}\right|=Z_{s} \wedge \frac{1}{(m-1) !} \omega^{m-1} .
$$

1.2. Random sections and Gaussian measures. We now give $H^{0}\left(M, L^{N}\right)$ the complex Gaussian probability measure

$$
d \mu(s)=\frac{1}{\pi^{d_{N}}} e^{-|c|^{2}} d c, \quad s=\sum_{j=1}^{d_{N}} c_{j} S_{j}^{N},
$$

where $\left\{S_{j}^{N}\right\}$ is an orthonormal basis for $H^{0}\left(M, L^{N}\right)$ and $d c$ is $2 d_{N^{-}}$dimensional Lebesgue measure. This Gaussian is characterized by the property that the $2 d_{N}$ real variables $\Re c_{j}$, $\Im c_{j}$ $\left(j=1, \ldots, d_{N}\right)$ are independent random variables with mean 0 and variance $\frac{1}{2}$; i.e.,

$$
\mathbf{E} c_{j}=0, \quad \mathbf{E} c_{j} c_{k}=0, \quad \mathbf{E} c_{j} \bar{c}_{k}=\delta_{j k} .
$$

Here and throughout this paper, $\mathbf{E}$ denotes expectation: $\mathbf{E} \varphi=\int \varphi d \mu$.

We then regard the currents $Z_{s}$ (resp. measures $\left|Z_{s}\right|$ ), as current-valued (resp. measurevalued) random variables on the probability space $\left(H^{0}\left(M, L^{N}\right), d \mu\right)$; i.e., for each test form (resp. function) $\varphi,\left(Z_{s}, \varphi\right)$ (resp. $\left.\left(\left|Z_{s}\right|, \varphi\right)\right)$ is a complex-valued random variable.

Since the zero current $Z_{s}$ is unchanged when $s$ is multiplied by an element of $\mathbb{C}^{*}$, our results are the same if we instead regard $Z_{s}$ as a random variable on the unit sphere $S H^{0}\left(M, L^{N}\right)$ 
with Haar probability measure. We prefer to use Gaussian measures in order to facilitate our computations.

1.3. Correlation currents. The $n$-point correlation current of the zeros is the current on $M^{n}=M \times M \times \cdots \times M$ ( $n$ times $)$ given by

$$
\vec{K}_{n}^{N}\left(z^{1}, \ldots, z^{n}\right):=\mathbf{E}\left(Z_{s}\left(z^{1}\right) \otimes Z_{s}\left(z^{2}\right) \otimes \cdots \otimes Z_{s}\left(z^{n}\right)\right)
$$

in the sense that for any test form $\varphi_{1}\left(z^{1}\right) \otimes \cdots \otimes \varphi_{n}\left(z^{n}\right) \in \mathcal{D}^{m-1, m-1}(M) \otimes \cdots \otimes \mathcal{D}^{m-1, m-1}(M)$,

$$
\left(\vec{K}_{n}^{N}\left(z^{1}, \ldots, z^{n}\right), \varphi_{1}\left(z^{1}\right) \otimes \cdots \otimes \varphi_{n}\left(z^{n}\right)\right)=\mathbf{E}\left[\left(Z_{s}, \varphi_{1}\right)\left(Z_{s}, \varphi_{2}\right) \cdots\left(Z_{s}, \varphi_{n}\right)\right] .
$$

In a similar way we define the $n$-point correlation measures $\widetilde{K}_{n}^{N}$ as the "total variation measures" of the $n$-point correlation currents:

$$
\widetilde{K}_{n}^{N}\left(z^{1}, \ldots, z^{n}\right)=\vec{K}_{n}^{N}\left(z^{1}, \ldots, z^{n}\right) \wedge \frac{1}{(m-1) !} \omega_{z^{1}}^{m-1} \wedge \cdots \wedge \frac{1}{(m-1) !} \omega_{z^{n}}^{m-1},
$$

i.e.,

$$
\left(\widetilde{K}_{n}^{N}\left(z^{1}, \ldots, z^{n}\right), \varphi_{1}\left(z^{1}\right) \ldots \varphi_{n}\left(z^{n}\right)\right)=\mathbf{E}\left[\left(\left|Z_{s}\right|, \varphi_{1}\right)\left(\left|Z_{s}\right|, \varphi_{2}\right) \cdots\left(\left|Z_{s}\right|, \varphi_{n}\right)\right]
$$

where $\varphi_{j} \in \mathcal{C}^{0}(M)$.

Remark: In the case of pair correlation on a Riemann surface $(n=2, \operatorname{dim} M=1)$, the correlation measures take the form

$$
\vec{K}_{2}^{N}(z, w)=[\Delta] \wedge\left(\vec{K}_{1}^{N}(z) \otimes 1\right)+\kappa^{N}(z, w) \omega_{z} \otimes \omega_{w} \quad(N \gg 0)
$$

where $[\Delta]$ denotes the current of integration along the diagonal $\Delta=\{(z, z)\} \subset M \times M$, and $\kappa^{N} \in \mathcal{C}^{\infty}(M \times M)$.

1.4. Szego kernels. As in [ZZ, SZ, BSZ] and elsewhere, we analyze the $N \rightarrow \infty$ limit by lifting it to a principal $S^{1}$ bundle $\pi: X \rightarrow M$. Let us recall how this goes.

We denote by $L^{*}$ the dual line bundle to $L$, and define $X$ as the circle bundle $X=\{\lambda \in$ $\left.L^{*}:\|\lambda\|_{h^{*}}=1\right\}$, where $h^{*}$ is the norm on $L^{*}$ dual to $h$. We can view $X$ as the boundary of the disc bundle $D=\left\{\lambda \in L^{*}: \rho(\lambda)>0\right\}$, where $\rho(\lambda)=1-\|\lambda\|_{h^{*}}^{2}$. The disc bundle $D$ is strictly pseudoconvex in $L^{*}$, since $\Theta_{h}$ is positive, and hence $X$ inherits the structure of a strictly pseudoconvex CR manifold. Associated to $X$ is the contact form $\alpha=-\left.i \partial \rho\right|_{X}=\left.i \bar{\partial} \rho\right|_{X}$. We also give $X$ the volume form

$$
d V_{X}=\frac{1}{m !} \alpha \wedge(d \alpha)^{m}=\alpha \wedge \pi^{*} d V_{M}
$$

The setting for our analysis of the Szegö kernel is the Hardy space $H^{2}(X) \subset \mathcal{L}^{2}(X)$ of square integrable $\mathrm{CR}$ functions on $X$, where we use the inner product

$$
\left\langle F_{1}, F_{2}\right\rangle=\frac{1}{2 \pi} \int_{X} F_{1} \overline{F_{2}} d V_{X}, \quad F_{1}, F_{2} \in \mathcal{L}^{2}(X) .
$$

We let $r_{\theta} x=e^{i \theta} x(x \in X)$ denote the $S^{1}$ action on $X$. The action $r_{\theta}$ commutes with the Cauchy-Riemann operator $\bar{\partial}_{b}$; hence $H^{2}(X)=\bigoplus_{N=0}^{\infty} H_{N}^{2}(X)$, where

$$
H_{N}^{2}(X)=\left\{F \in H^{2}(X): F\left(r_{\theta} x\right)=e^{i N \theta} F(x)\right\} .
$$


A section $s_{N}$ of $L^{N}$ determines an equivariant function $\hat{s}_{N}$ on $X$ :

$$
\hat{s}_{N}(z, \lambda)=\left(\lambda^{\otimes N}, s_{N}(z)\right), \quad(z, \lambda) \in X ;
$$

then $\hat{s}_{N}\left(r_{\theta} x\right)=e^{i N \theta} s_{N}(x)$. The map $s \mapsto \hat{s}$ is a unitary equivalence between $H^{0}\left(M, L^{\otimes N}\right)$ and $H_{N}^{2}(X)$.

We let $\Pi_{N}: \mathcal{L}^{2}(X) \rightarrow H_{N}^{2}(X)$ denote the orthogonal projection. The Szegö kernel $\Pi_{N}(x, y)$ is defined by

$$
\Pi_{N} F(x)=\int_{X} \Pi_{N}(x, y) F(y) d V_{X}(y), \quad F \in \mathcal{L}^{2}(X)
$$

It can be given as

$$
\Pi_{N}(x, y)=\sum_{j=1}^{d_{N}} \widehat{S}_{j}^{N}(x) \overline{\widehat{S}_{j}^{N}(y)}
$$

where $S_{1}^{N}, \ldots, S_{d_{N}}^{N}$ form an orthonormal basis of $H^{0}\left(M, L^{N}\right)$.

\section{SCALING}

In order that we may study the local nature of the random variable $Z_{s}$, we fix a point $z^{0} \in M$ and choose a holomorphic coordinate chart $\Psi: \Omega, 0 \rightarrow U, z_{0}\left(\Omega \subset \mathbb{C}^{m}, U \subset M\right)$ such that

$$
\Psi^{*} \omega_{z^{0}}=\left.\frac{i}{2} \sum_{j=1}^{m} d z_{j} \wedge d \bar{z}_{j}\right|_{0}
$$

For example, if $L$ is the hyperplane section bundle $\mathcal{O}(1)$ over $\mathbb{C P}^{m}$ with the Fubini-Study metric $h_{\mathrm{FS}}$, and $z_{0}=(1: 0: \cdots: 0)$, then the coordinate chart

$$
\Psi: \mathbb{C}^{m} \rightarrow U=\left\{w \in \mathbb{C P}^{m}: w_{0} \neq 0\right\}, \quad \Psi(z)=\left(1: z_{1}: \cdots: z_{m}\right)
$$

(i.e., $\left.z_{j}=w_{j} / w_{0}\right)$ satisfies (17).

To simplify notation, we identify $U$ with $\Omega$. For a current $T \in \mathcal{D}^{\prime p, q}(\Omega)$, we write

$$
T\left(\frac{z}{\sqrt{N}}\right)=\left(\tau_{\sqrt{N}}\right)_{*} T \in \mathcal{D}^{\prime p, q}(\sqrt{N} \Omega) \quad\left(\tau_{\lambda}(z)=\lambda z\right) .
$$

(In particular, if $T=\sum T_{j k}(z) d z_{j} \wedge d \bar{z}_{k}$, then $T\left(\frac{z}{\sqrt{N}}\right)=\frac{1}{N} \sum T_{j k}\left(\frac{z}{\sqrt{N}}\right) d z_{j} \wedge d \bar{z}_{k}$.)

We define the rescaled zero current of $s \in H^{0}\left(M, L^{N}\right)$ by

$$
\widehat{Z}_{s}^{N}(z):=Z_{s}\left(\frac{z}{\sqrt{N}}\right) .
$$

The scaled $n$-point correlation currents are then defined by:

$$
\mathbf{E}\left(\widehat{Z}_{s}^{N}\left(z^{1}\right) \otimes \widehat{Z}_{s}^{N}\left(z^{2}\right) \otimes \cdots \otimes \widehat{Z}_{s}^{N}\left(z^{n}\right)\right)=\vec{K}_{n}^{N}\left(\frac{z^{1}}{\sqrt{N}}, \ldots, \frac{z^{n}}{\sqrt{N}}\right) \in \mathcal{D}^{\prime n, n}\left(M^{n}\right) .
$$


Following the approach of [SZ], we fix an orthonormal basis $\left\{S_{j}^{N}\right\}$ of $H^{0}\left(M, L^{N}\right)$ and write $S_{j}^{N}=f_{j}^{N} e_{L}^{\otimes N}$ over $U$. Any section in $H^{0}\left(M, L^{N}\right)$ may then be written as $s=\sum_{j=1}^{d_{N}} c_{j} f_{j}^{N} e_{L}^{N}$. To simplify the notation we let $f^{N}=\left(f_{1}^{N}, \ldots, f_{d_{N}}^{N}\right): U \rightarrow \mathbb{C}^{d_{N}}$ and we put

$$
\sum_{j=1}^{d_{N}} c_{j} f_{j}=c \cdot f^{N}
$$

Hence

$$
Z_{s}=\frac{\sqrt{-1}}{\pi} \partial \bar{\partial} \log \left|c \cdot f^{N}\right|, \quad \widehat{Z}_{s}^{N}=\frac{\sqrt{-1}}{\pi} \partial_{z} \bar{\partial}_{z} \log \left|c \cdot f^{N}\left(\frac{z}{\sqrt{N}}\right)\right|
$$

and therefore

$$
\widehat{Z}_{s}\left(z^{1}\right) \otimes \cdots \otimes \widehat{Z}_{s}\left(z^{n}\right)=\left(\frac{i}{\pi}\right)^{n} \partial_{z^{1}} \bar{\partial}_{z^{1}} \cdots \partial_{z^{n}} \bar{\partial}_{z^{n}}\left[\log \left|c \cdot f^{N}\left(\frac{z^{1}}{\sqrt{N}}\right)\right| \cdots \log \left|c \cdot f^{N}\left(\frac{z^{n}}{\sqrt{N}}\right)\right|\right] .
$$

We then can write the rescaled correlation currents in the form

$$
\begin{aligned}
& \vec{K}_{n}^{N}\left(\frac{z^{1}}{\sqrt{N}}, \ldots, \frac{z^{n}}{\sqrt{N}}\right)=\mathbf{E}\left(\widehat{Z}_{s}\left(z^{1}\right) \otimes \cdots \otimes \widehat{Z}_{s}\left(z^{n}\right)\right) \\
& \quad=\left(\frac{i}{\pi}\right)^{n} \partial_{z^{1}} \bar{\partial}_{z^{1}} \cdots \partial_{z^{n}} \bar{\partial}_{z^{n}} \int_{\mathbb{C}^{d_{N}}} \log \left|c \cdot f^{N}\left(\frac{z^{1}}{\sqrt{N}}\right)\right| \cdots \log \left|c \cdot f^{N}\left(\frac{z^{n}}{\sqrt{N}}\right)\right| \frac{e^{-|c|^{2}}}{\pi^{d_{N}}} d c .
\end{aligned}
$$

2.1. Scaling limit of the Szego kernel. The asymptotics of the Szegö kernel along the diagonal were given by [Ti] and [Ze]:

$$
\frac{\pi^{m}}{N^{m}} \Pi_{N}(x, x)=1+O\left(N^{-1}\right) .
$$

For our proof of the Main Theorem, we need the following lemma from [BSZ], which gives the 'near-diagonal' asymptotics of the Szego kernel.

LEMMA 2.1. Let $z^{0} \in M$ and choose local coordinates $\left\{z^{j}\right\}$ in a neighborhood of $z_{0}$ so that $z^{0}=0$ and $\Theta_{h}\left(z_{0}\right)=\sum d z^{j} \wedge d \bar{z}^{j}$. Then

$$
\frac{\pi^{m}}{N^{m}} \Pi_{N}\left(\frac{z}{\sqrt{N}}, \frac{\theta}{N} ; \frac{w}{\sqrt{N}}, \frac{\varphi}{N}\right)=e^{i 2 \pi(\theta-\varphi)+i \Im(z \cdot \bar{w})-\frac{1}{2}|z-w|^{2}}+O\left(N^{-1 / 2}\right) .
$$

Here, $(z, \theta)$ denotes the point $e^{i \theta}\left\|e_{L}(z)\right\|_{h} e_{L}^{*}(z) \in X$, and similarly for $(w, \varphi)$. In (23) and Lemma 2.1, the expression $O\left(N^{\alpha}\right)$ means a term with $k^{\text {th }}$ order derivatives $\leq C_{k} N^{\alpha}$, for all $k \geq 0$. Lemma 2.1 says that the Szegö kernel has a universal scaling limit. In fact, its scaling limit is the first Szegö kernel of the reduced Heisenberg group; see BSZ.

\section{UNIVERSALITY}

All the ideas of the proof of the Main Theorem occur in the simplest case $n=2$. So first we prove universality in that case and then extend the proof to general $n$. 
Thus, our first object is to prove that the large $N$ limit of the rescaled pair correlation current (from (22) with $n=2$ )

$$
\begin{aligned}
& \vec{K}_{2}^{N}\left(\frac{z}{\sqrt{N}}, \frac{w}{\sqrt{N}}\right)=\mathbf{E}\left(\widehat{Z}_{s}^{N}(z) \otimes \widehat{Z}_{s}^{N}(w)\right) \\
& \quad=\frac{-1}{\pi^{2}} \partial_{z} \bar{\partial}_{z} \partial_{w} \bar{\partial}_{w} \int_{\mathbb{C}^{d_{N}}} \log \left|c \cdot f^{N}\left(\frac{z}{\sqrt{N}}\right)\right| \log \left|c \cdot f^{N}\left(\frac{w}{\sqrt{N}}\right)\right| \frac{e^{-|c|^{2}}}{\pi^{d_{N}}} d c
\end{aligned}
$$

is universal.

As in [SZ], we write $f^{N}=\left|f^{N}\right| u^{N}$ and expand the integrand in (24):

$$
\begin{aligned}
\log \left|c \cdot f^{N}\left(\frac{z}{\sqrt{N}}\right)\right| \log \left|c \cdot f^{N}\left(\frac{w}{\sqrt{N}}\right)\right|= & \log \left|f^{N}\left(\frac{z}{\sqrt{N}}\right)\right| \log \left|f^{N}\left(\frac{w}{\sqrt{N}}\right)\right| \\
& +\log \left|f^{N}\left(\frac{z}{\sqrt{N}}\right)\right| \log \left|c \cdot u^{N}\left(\frac{w}{\sqrt{N}}\right)\right| \\
& +\log \left|f^{N}\left(\frac{w}{\sqrt{N}}\right)\right| \log \left|c \cdot u^{N}\left(\frac{z}{\sqrt{N}}\right)\right| \\
& +\log \left|c \cdot u^{N}\left(\frac{z}{\sqrt{N}}\right)\right| \log \left|c \cdot u^{N}\left(\frac{w}{\sqrt{N}}\right)\right| .
\end{aligned}
$$

Let us denote the terms resulting from this expansion by $E_{1}, E_{2}, E_{3}, E_{4}$, respectively. In particular,

$$
E_{1}=\frac{-1}{\pi^{2}} \partial_{z} \bar{\partial}_{z} \partial_{w} \bar{\partial}_{w}\left[\log \left|f^{N}\left(\frac{z}{\sqrt{N}}, \frac{z}{\sqrt{N}}\right)\right| \log \left|f^{N}\left(\frac{w}{\sqrt{N}}, \frac{w}{\sqrt{N}}\right)\right|\right] .
$$

By (14), $\widehat{S}_{j}^{N}(z, \theta)=e^{i N \theta}\left\|e_{L}(z)\right\|_{h}^{N} f_{j}^{N}(z)$, where $(z, \theta)$ are the coordinates in $X$ given in 2.1. By $(16)$,

$$
\Pi_{N}(z, w)=\left\|e_{L}(z)\right\|_{h}^{N}\left\|e_{L}(w)\right\|_{h}^{N}\left\langle f^{N}(z), f^{N}(w)\right\rangle,
$$

where we write $\Pi_{N}(z, w)=\Pi_{N}(z, 0 ; w, 0)$. Since $\Pi_{N}(z, z)^{1 / 2}=\left\|e_{L}(z)\right\|_{h}^{N}\left|f^{N}(z)\right|$, each factor in (26) has the form $\frac{1}{2} \log \Pi_{N}\left(\frac{z}{\sqrt{N}}, \frac{z}{\sqrt{N}}\right)-N \log \left\|e_{L}\left(\frac{z}{\sqrt{N}}\right)\right\|_{h}$. By (23), $\log \Pi_{N}\left(\frac{z}{\sqrt{N}}, \frac{z}{\sqrt{N}}\right) \rightarrow 0$ as $N \rightarrow \infty$. On the other hand

$$
-i N \partial_{z} \bar{\partial}_{z} \log \left\|e_{L}\left(\frac{z}{\sqrt{N}}\right)\right\|_{h}=\omega\left(\frac{z}{\sqrt{N}}\right) .
$$

Hence the first term converges to the normalized Euclidean (double) Kähler form:

$$
E_{1}=\frac{i}{2 \pi} \partial \bar{\partial}|z|^{2} \wedge \frac{i}{2 \pi} \partial \bar{\partial}|w|^{2}+O\left(\frac{1}{N}\right) .
$$

The middle two terms vanish since the integrals in $E_{2}$ and $E_{3}$ are independent of $w$ and $z$ respectively (see [SZ, §3.2]). The "interesting term" is therefore

$$
E_{4}=\frac{-1}{\pi^{2}} \partial_{z} \bar{\partial}_{z} \partial_{w} \bar{\partial}_{w} \int_{\mathbb{C}^{d_{N}}} \log \left|c \cdot u^{N}\left(\frac{z}{\sqrt{N}}\right)\right| \log \left|c \cdot u^{N}\left(\frac{w}{\sqrt{N}}\right)\right| \frac{e^{-|c|^{2}}}{\pi^{d_{N}}} d c .
$$

To evaluate $E_{4}$, we consider the integral

$$
G_{2}^{N}\left(x^{1}, x^{2}\right):=\int_{\mathbb{C}^{d_{N}}} \log \left|c \cdot x^{1}\right| \log \left|c \cdot x^{2}\right| \frac{e^{-|c|^{2}}}{\pi^{d_{N}}} d c \quad\left(x^{1}, x^{2} \in \mathbb{C}^{d_{N}}\right)
$$


with $x^{1}=u^{N}\left(\frac{z}{\sqrt{N}}\right), x^{2}=u^{N}\left(\frac{w}{\sqrt{N}}\right)$. To simplify it, we construct a Hermitian orthonormal basis $\left\{e_{1}, \ldots, e_{d_{N}}\right\}$ for $\mathbb{C}^{d_{N}}$ such that $x^{1}=e_{1}$ and

$$
x^{2}=\xi_{1} e_{1}+\xi_{2} e_{2}, \quad \xi_{1}=\left\langle x^{2}, x^{1}\right\rangle, \quad \xi_{2}=\sqrt{1-\left|\xi_{1}\right|^{2}}
$$

This is possible because we can always multiply $e_{2}$ by a phase $e^{i \theta}$ so that $\xi_{2}$ is positive real. We then make a unitary change of variables to express the integral in the $\left\{e_{j}\right\}$ coordinates. Since the Gaussian is $U\left(d_{N}\right)$-invariant, (30) simplifies to

$$
G_{2}^{N}\left(x^{1}, x^{2}\right)=G_{2}\left(\xi_{1}, \xi_{2}\right)=\frac{1}{\pi^{2}} \int_{\mathbb{C}^{2}} e^{-\left(\left|c_{1}\right|^{2}+\left|c_{2}\right|^{2}\right)} \log \left|\xi_{1}\right| \log \left|c_{1} \xi_{1}+c_{2} \xi_{2}\right| d c_{1} d c_{2}
$$

(where we used the fact that the Gaussian integral in each $c_{j}, j \geq 3$ equals one by construction). By performing a rotation of the $c_{1}$ variable, we may replace $\xi_{1}$ with $\left|\xi_{1}\right|$ and replace $G_{2}\left(\xi_{1}, \xi_{2}\right)$ with

$$
G(\cos \theta):=G_{2}(\cos \theta, \sin \theta),
$$

where $\cos \theta=\left|\xi_{1}\right|=\left|\left\langle x^{1}, x^{2}\right\rangle\right|, 0 \leq \theta \leq \pi / 2$. Hence (29) becomes

$$
E_{4}=\frac{-1}{\pi^{2}} \partial_{z} \bar{\partial}_{z} \partial_{w} \bar{\partial}_{w} G\left(\cos \theta_{N}\right), \quad \cos \theta_{N}=\left|\left\langle u^{N}\left(\frac{z}{\sqrt{N}}\right), u^{N}\left(\frac{w}{\sqrt{N}}\right)\right\rangle\right| .
$$

By the universal scaling formula for the Szego kernel (Lemma 2.1) and (27), we have

$$
\cos \theta_{N}=\frac{\left|\Pi_{N}(z, w)\right|}{\Pi_{N}(z, z)^{1 / 2} \Pi_{N}(w, w)^{1 / 2}}=e^{-\frac{1}{2}|z-w|^{2}}+O\left(N^{-\frac{1}{2}}\right) .
$$

Thus we get the universal formula:

$$
\vec{K}_{2}^{\infty}(z, w)=\frac{i}{2 \pi} \partial \bar{\partial}|z|^{2} \wedge \frac{i}{2 \pi} \partial \bar{\partial}|w|^{2}+\frac{-1}{\pi^{2}} \partial_{z} \bar{\partial}_{z} \partial_{w} \bar{\partial}_{w} G\left(e^{-\frac{1}{2}|z-w|^{2}}\right) .
$$

This completes the proof for the pair correlation case $n=2$. (Notice that the formula has the same form in all dimensions.)

The proof for general $n$ is similar. We again write $f^{N}=\left|f^{N}\right| u^{N}$ and expand the integrand in (22):

$$
\begin{aligned}
& \log \left|c \cdot f^{N}\left(\frac{z^{1}}{\sqrt{N}}\right)\right| \log \left|c \cdot f^{N}\left(\frac{z^{2}}{\sqrt{N}}\right)\right| \cdots \log \left|c \cdot f^{N}\left(\frac{z^{n}}{\sqrt{N}}\right)\right| \\
& =\log \left|f^{N}\left(\frac{z^{1}}{\sqrt{N}}\right)\right| \log \left|f^{N}\left(\frac{z^{2}}{\sqrt{N}}\right)\right| \cdots \log \left|f^{N}\left(\frac{z^{n}}{\sqrt{N}}\right)\right| \\
& +\log \left|f^{N}\left(\frac{z^{1}}{\sqrt{N}}\right)\right| \log \left|f^{N}\left(\frac{z^{2}}{\sqrt{N}}\right)\right| \cdots \log \left|f^{N}\left(\frac{z^{n-1}}{\sqrt{N}}\right)\right| \log \left|c \cdot u^{N}\left(\frac{z^{n}}{\sqrt{N}}\right)\right| \\
& +\cdots \\
& +\log \left|c \cdot u^{N}\left(\frac{z^{1}}{\sqrt{N}}\right)\right| \log \left|c \cdot u^{N}\left(\frac{z^{2}}{\sqrt{N}}\right)\right| \cdots \log \left|c \cdot u^{N}\left(\frac{z^{n}}{\sqrt{N}}\right)\right| .
\end{aligned}
$$

We denote the terms resulting from this expansion by $E_{1}, \ldots, E_{2^{n}}$, respectively. As before, the first term converges to the normalized Euclidean " $n$-fold" Kähler form:

$$
E_{1}=\frac{i}{2 \pi} \partial \bar{\partial}\left|z^{1}\right|^{2} \wedge \cdots \wedge \frac{i}{2 \pi} \partial \bar{\partial}\left|z^{n}\right|^{2}+O\left(\frac{1}{N}\right) .
$$


The $E_{2^{n}}$ term is obtained from the function

$$
G_{n}^{N}\left(x^{1}, x^{2}, \ldots, x^{n}\right):=\int_{\mathbb{C}^{d_{N}}} \log \left|c \cdot x^{1}\right| \log \left|c \cdot x^{2}\right| \cdots \log \left|c \cdot x^{n}\right| \frac{e^{-|c|^{2}}}{\pi^{d_{N}}} d c,
$$

$x^{1}, x^{2}, \ldots, x^{n} \in \mathbb{C}^{d_{N}}$. Precisely, we substitute

$$
x^{j}=u^{N}\left(\frac{z^{j}}{\sqrt{N}}\right)
$$

in (37) and apply the operator $\left(\frac{i}{\pi}\right)^{n} \partial_{z^{1}} \bar{\partial}_{z^{1}} \cdots \partial_{z^{n}} \bar{\partial}_{z^{n}}$. As above, we define a special Hermitian orthonormal basis $\left\{e_{1}, \ldots, e_{n}\right\}$ for the n-dimensional complex subspace spanned by $\left\{x_{1}, \ldots, x_{n}\right\}$. We put:

$$
\begin{array}{ll}
x^{1}=e_{1} & \xi_{22}=\sqrt{1-\left|\xi_{21}\right|^{2}} \\
x^{2}=\xi_{21} e_{1}+\xi_{22} e_{2} & \\
\vdots & \xi_{n n}=\sqrt{1-\sum_{j \leq n-1}\left|\xi_{n j}\right|^{2}} . \\
x^{n}=\xi_{n 1} e_{1}+\cdots+\xi_{n n} e_{n} &
\end{array}
$$

Such a basis exists because we can always multiply $e_{j}$ by a phase $e^{i \theta}$ so that the last component $\xi_{j j}$ is positive real. We complete $\left\{e_{j}\right\}$ to a basis of $\mathbb{C}^{d_{N}}$, and we now let $c_{j}$ denote coordinates relative to this basis. As above, we rewrite the Gaussian integral in these coordinates. After integrating out the variables $\left\{c_{n+1}, \ldots, c_{d_{N}}\right\}$, (37) simplifies to the $n$-dimensional complex Gaussian integral

$$
\begin{aligned}
G_{n}^{N}\left(x^{1}, \ldots, x^{n}\right) & =G_{n}\left(\xi_{21}, \xi_{22}, \ldots, \xi_{n n}\right) \\
& =\frac{1}{\pi^{n}} \int_{\mathbb{C}^{n}} e^{-|c|^{2}} \log \left|c_{1}\right| \log \left|c_{1} \xi_{21}+c_{2} \xi_{22}\right| \cdots \log \left|c_{1} \xi_{n 1}+\ldots c_{n} \xi_{n n}\right| d c .
\end{aligned}
$$

Note that the variables $\xi_{j k}$ depend on $N$; we write $\xi_{j k}=\xi_{j k}^{N}$ when we need to indicate this dependence.

To prove universality, we observe that the $\xi_{j k}$ are universal algebraic functions of the inner products $\left\langle x^{a}, x^{b}\right\rangle$. Indeed,

$$
\xi_{j 1} \bar{\xi}_{k 1}+\cdots+\xi_{j k} \bar{\xi}_{k k}=\left\langle x^{j}, x^{k}\right\rangle, \quad 1 \leq k \leq j \leq n
$$

where we set $\xi_{11}=1$. These algebraic functions are obtained by induction (lexicographically) using (40). (The triangular matrix $\left(\xi_{j k}\right)$ is just the inverse of the matrix describing the GramSchmidt process.)

By (38), it follows that the $\xi_{j k}^{N}$ are universal algebraic functions of the variables

$$
\left\langle u^{N}\left(\frac{z^{j}}{\sqrt{N}}\right), u^{N}\left(\frac{z^{k}}{\sqrt{N}}\right)\right\rangle=\frac{\Pi_{N}\left(\frac{z^{j}}{\sqrt{N}}, \frac{z^{k}}{\sqrt{N}}\right)}{\prod_{N}\left(\frac{z^{j}}{\sqrt{N}}, \frac{z^{j}}{\sqrt{N}}\right)^{1 / 2} \Pi_{N}\left(\frac{z^{k}}{\sqrt{N}}, \frac{z^{k}}{\sqrt{N}}\right)^{1 / 2}}=e^{i \Im\left(z^{j} \cdot \bar{z}^{k}\right)-\frac{1}{2}\left|z^{j}-z^{k}\right|^{2}}+O\left(\frac{1}{\sqrt{N}}\right) .
$$

We note here that

$$
\left|x^{1} \wedge \cdots \wedge x^{n}\right|^{2}=\operatorname{det}\left(\left\langle x^{j}, x^{k}\right\rangle\right) \rightarrow \operatorname{det}\left(e^{i \Im\left(z^{j} \cdot \bar{z}^{k}\right)-\frac{1}{2}\left|z^{j}-z^{k}\right|^{2}}\right)=e^{-\sum\left|z^{j}\right|^{2}} \operatorname{det}\left(e^{z_{j} \cdot \bar{z}_{k}}\right) .
$$


When the $z_{j}$ are distinct (i.e., $\left.\left(z^{1}, \ldots, z^{n}\right) \in \mathcal{G}_{n}^{m}\right)$, the limit determinant in (41) is nonzero (see [BSZ]) and thus $\xi_{j k}^{N}=\xi_{j k}^{\infty}+O\left(\frac{1}{\sqrt{N}}\right)$, where the $\xi_{j k}^{\infty}$ are universal real-analytic functions of $z \in \mathcal{G}_{n}^{m}$. We conclude that the $E_{2^{n}}$ term converges to a universal current:

$$
E_{2^{n}}=\left(\frac{i}{\pi}\right)^{n} \partial_{z^{1}} \bar{\partial}_{z^{1}} \cdots \partial_{z^{n}} \bar{\partial}_{z^{n}} G_{n}\left(\xi_{21}^{\infty}, \ldots, \xi_{k k}^{\infty}\right)+O\left(\frac{1}{\sqrt{N}}\right)
$$

Consider now a general term $E_{a}$. Suppose without loss of generality that $E_{a}$ comes from

$$
\log \left|c \cdot u^{N}\left(\frac{z^{1}}{\sqrt{N}}\right)\right| \cdots \log \left|c \cdot u^{N}\left(\frac{z^{k}}{\sqrt{N}}\right)\right| \log \left|f^{N}\left(\frac{z^{k+1}}{\sqrt{N}}\right)\right| \cdots \log \left|f^{N}\left(\frac{z^{n}}{\sqrt{N}}\right)\right| .
$$

As above we obtain

$$
E_{a}=\left(\frac{i}{\pi}\right)^{k} \partial_{z^{1}} \bar{\partial}_{z^{1}} \cdots \partial_{z^{k}} \bar{\partial}_{z^{k}} G_{k}\left(\xi_{21}^{\infty}, \ldots, \xi_{k k}^{\infty}\right) \wedge \frac{i}{2 \pi} \partial \bar{\partial}\left|z^{k+1}\right|^{2} \wedge \cdots \wedge \frac{i}{2 \pi} \partial \bar{\partial}\left|z^{n}\right|^{2}+O\left(\frac{1}{\sqrt{N}}\right) .
$$

Hence this term also approaches a universal current. (As in the pair correlation case, terms with only one $u^{N}$ vanish.)

\section{EXPLiCIT FORMULAE}

We now calculate explicitly the limit pair correlation measures $\widetilde{K}_{2}^{\infty}(z, w)$.

4.1. Preliminaries. The first step is to compute $\Delta G\left(e^{-\frac{1}{2} r^{2}}\right)$, where $\Delta$ is the Euclidean Laplacian on $\mathbb{C}^{m}$ and $r=|\zeta|\left(\zeta \in \mathbb{C}^{m}\right)$. To begin this computation, we write $a_{j}=r_{j} e^{i \varphi_{j}}$ and then rewrite (32)-(33) as

$$
G(\cos \theta)=\frac{2}{\pi} \int_{0}^{\infty} \int_{0}^{\infty} \int_{0}^{2 \pi} r_{1} r_{2} e^{-\left(r_{1}^{2}+r_{2}^{2}\right)} \log r_{1} \log \left|r_{1} \cos \theta+r_{2} e^{i \varphi} \sin \theta\right| d \varphi d r_{1} d r_{2} .
$$

We now evaluate the inner integral by Jensen's formula, which gives

$$
\int_{0}^{2 \pi} \log \left|r_{1} \cos \theta+r_{2} \sin \theta e^{i \varphi}\right| d \varphi= \begin{cases}2 \pi \log \left(r_{1} \cos \theta\right) & \text { for } r_{2} \sin \theta \leq r_{1} \cos \theta \\ 2 \pi \log \left(r_{2} \sin \theta\right) & \text { for } r_{2} \sin \theta \geq r_{1} \cos \theta\end{cases}
$$

Hence

$$
G(\cos \theta)=4 \int_{0}^{\infty} \int_{0}^{\infty} r_{1} r_{2} e^{-\left(r_{1}^{2}+r_{2}^{2}\right)} \log r_{1} \log \max \left(r_{1} \cos \theta, r_{2} \sin \theta\right) d r_{1} d r_{2} .
$$

Now change variables again with $r_{1}=\rho \cos \varphi, r_{2}=\rho \sin \varphi$ to get

$$
G(\cos \theta)=4 \int_{0}^{\infty} \int_{0}^{\pi / 2} \rho^{3} e^{-\rho^{2}} \log (\rho \cos \varphi) \log \max (\rho \cos \varphi \cos \theta, \rho \sin \varphi \sin \theta) \cos \varphi \sin \varphi d \varphi d \rho .
$$

Since

$$
\log \max (\rho \cos \varphi \cos \theta, \rho \sin \varphi \sin \theta)=\log (\rho \cos \varphi \cos \theta)+\log ^{+}(\tan \varphi \tan \theta)
$$


we can write $G=G_{1}+G_{2}$, where

$$
\begin{aligned}
& G_{1}(\cos \theta)=4 \int_{0}^{\infty} \int_{0}^{\pi / 2} \rho^{3} e^{-\rho^{2}} \log (\rho \cos \varphi) \log (\rho \cos \varphi \cos \theta) \cos \varphi \sin \varphi d \varphi d \rho \\
& G_{2}(\cos \theta)=4 \int_{0}^{\infty} \int_{\pi / 2-\theta}^{\pi / 2} \rho^{3} e^{-\rho^{2}} \log (\rho \cos \varphi) \log (\tan \varphi \tan \theta) \cos \varphi \sin \varphi d \varphi d \rho .
\end{aligned}
$$

From (46), $G_{1}(\cos \theta)=C_{1}+C_{2} \log \cos \theta$ and thus

$$
G_{1}\left(e^{-\frac{1}{2} r^{2}}\right)=C_{1}-\frac{1}{2} C_{2} r^{2}
$$

so that

$$
\Delta G_{1}\left(e^{-\frac{1}{2} r^{2}}\right)=\left(\frac{d^{2}}{d r^{2}}+\frac{2 m-1}{r} \frac{d}{d r}\right)\left(C_{1}-\frac{1}{2} C_{2} r^{2}\right)=-2 m C_{2} .
$$
have

We now evaluate $\Delta G_{2}\left(e^{-\frac{1}{2} r^{2}}\right)$. Since the integrand in (47) vanishes when $\varphi=\pi / 2-\theta$, we

$$
\frac{d}{d r} G_{2}(\cos \theta)=4\left(\frac{d}{d r} \log \tan \theta\right) \int_{0}^{\infty} \int_{\pi / 2-\theta}^{\pi / 2} \rho^{3} e^{-\rho^{2}} \log (\rho \cos \varphi) \cos \varphi \sin \varphi d \varphi d \rho .
$$

Substituting $\tan ^{2} \theta=e^{r^{2}}-1$, we have

$$
\frac{d}{d r} \log \tan \theta=\frac{r}{1-e^{-r^{2}}} .
$$

Thus

where

$$
\frac{d}{d r} G_{2}\left(e^{-\frac{1}{2} r^{2}}\right)=\frac{4 r}{1-e^{-r^{2}}}\left(I_{1}+I_{2}\right),
$$

$$
\begin{gathered}
I_{1}=\int_{0}^{\infty} \int_{\pi / 2-\theta}^{\pi / 2} \rho^{3} e^{-\rho^{2}}(\log \rho) \cos \varphi \sin \varphi d \varphi d \rho=C \sin ^{2} \theta=C\left(1-e^{-r^{2}}\right), \\
I_{2}=\int_{0}^{\infty} \int_{\pi / 2-\theta}^{\pi / 2} \rho^{3} e^{-\rho^{2}}(\log \cos \varphi) \cos \varphi \sin \varphi d \varphi d \rho .
\end{gathered}
$$

We compute

$$
\begin{aligned}
I_{2} & =\frac{1}{2} \int_{\pi / 2-\theta}^{\pi / 2}(\log \cos \varphi) \cos \varphi \sin \varphi d \varphi=\frac{1}{2} \int_{0}^{\sin \theta} t \log t d t \\
& =\frac{1}{8}\left(\sin ^{2} \theta \log \sin ^{2} \theta-\sin ^{2} \theta\right)=\frac{1}{8}\left(1-e^{-r^{2}}\right)\left[\log \left(1-e^{-r^{2}}\right)-1\right]
\end{aligned}
$$

Thus

$$
\frac{d}{d r} G_{2}\left(e^{-\frac{1}{2} r^{2}}\right)=\frac{r}{2} \log \left(1-e^{-r^{2}}\right)+C^{\prime} r
$$

Hence by (48) and (49),

$$
\begin{aligned}
\Delta G\left(e^{-\frac{1}{2} r^{2}}\right) & =-2 m C_{2}+\left(\frac{d}{d r}+\frac{2 m-1}{r}\right)\left(\frac{r}{2} \log \left(1-e^{-r^{2}}\right)+C^{\prime} r\right) \\
& =m \log \left(1-e^{-r^{2}}\right)+\frac{r^{2}}{e^{r^{2}}-1}+C^{\prime \prime} .
\end{aligned}
$$


4.2. Pair correlation in dimension 1. In dimension one, the pair correlation form is the same as the pair correlation measure. We first give our universal formula in the onedimensional case. Our formula agrees with that of Bogomolny-Bohigas-Leboeuf [BBL] and Hannay [Ha for $\mathrm{SU}(2)$ polynomials.

TheOREM 4.1. Suppose $\operatorname{dim} M=1$. Then

$$
\vec{K}_{2}^{N}\left(\frac{z}{\sqrt{N}}, \frac{w}{\sqrt{N}}\right) \rightarrow \vec{K}_{2}^{\infty}(z, w)=\left[\pi \delta_{0}(z-w)+H\left(\frac{1}{2}|z-w|^{2}\right)\right] \frac{i}{2 \pi} \partial \bar{\partial}|z|^{2} \wedge \frac{i}{2 \pi} \partial \bar{\partial}|w|^{2},
$$

where

$$
H(t)=\frac{\left(\sinh ^{2} t+t^{2}\right) \cosh t-2 t \sinh t}{\sinh ^{3} t}=t-\frac{2}{9} t^{3}+\frac{2}{45} t^{5}+O\left(t^{7}\right) .
$$

Proof. Making the change of variables $\zeta=z-w$, we have by (36),

$$
\begin{aligned}
\mathbf{E}\left(\widehat{Z}^{N}(z) \otimes \widehat{Z}^{N}(w)\right) \rightarrow & \frac{i}{2 \pi} \partial \bar{\partial}|z|^{2} \wedge \frac{i}{2 \pi} \partial \bar{\partial}|w|^{2}-\frac{1}{\pi^{2}} \partial_{z} \bar{\partial}_{z} \partial_{w} \bar{\partial}_{w} G\left(e^{-\frac{1}{2}|z-w|^{2}}\right) \\
& =\left[1+4 \frac{\partial^{2}}{\partial z \bar{\partial} z} \frac{\partial^{2}}{\partial w \bar{\partial} w} G\left(e^{-\frac{1}{2}|z-w|^{2}}\right)\right] \frac{i}{2 \pi} \partial \bar{\partial}|z|^{2} \wedge \frac{i}{2 \pi} \partial \bar{\partial}|w|^{2} \\
& =\left[1+4\left(\frac{\partial^{2}}{\partial \zeta \bar{\partial} \zeta}\right)^{2} G\left(e^{-\frac{1}{2}|\zeta|^{2}}\right)\right] \frac{i}{2 \pi} \partial \bar{\partial}|z|^{2} \wedge \frac{i}{2 \pi} \partial \bar{\partial}|w|^{2} \\
& =\left[1+\frac{1}{4} \Delta^{2} G\left(e^{-\frac{1}{2} r^{2}}\right)\right] \frac{i}{2 \pi} \partial \bar{\partial}|z|^{2} \wedge \frac{i}{2 \pi} \partial \bar{\partial}|w|^{2}
\end{aligned}
$$

By (50) with $m=1$, we have

$$
\begin{aligned}
\Delta^{2} G\left(e^{-\frac{1}{2} r^{2}}\right) & =\left(\frac{d^{2}}{d r^{2}}+\frac{1}{r} \frac{d}{d r}\right)\left[\log \left(1-e^{-r^{2}}\right)+\frac{r^{2}}{e^{r^{2}}-1}\right] \\
& =4 \pi \delta_{0}+\frac{8\left(e^{r^{2}}-1\right)^{2}-16 r^{2} e^{r^{2}}\left(e^{r^{2}}-1\right)+4 r^{4} e^{r^{2}}\left(e^{r^{2}}+1\right)}{\left(e^{r^{2}}-1\right)^{3}} .
\end{aligned}
$$

Finally,

$$
\begin{aligned}
{\left[1+\frac{1}{4} \Delta^{2} G\left(e^{-\frac{1}{2} r^{2}}\right)\right] } & =\pi \delta_{0}+\frac{\left(e^{r^{2}}+1\right)\left(e^{r^{2}}-1\right)^{2}-4 r^{2} e^{r^{2}}\left(e^{r^{2}}-1\right)+r^{4} e^{r^{2}}\left(e^{r^{2}}+1\right)}{\left(e^{r^{2}}-1\right)^{3}} \\
& =\pi \delta_{0}+\frac{\left(\sinh ^{2} \frac{1}{2} r^{2}+\frac{1}{4} r^{4}\right) \cosh \frac{1}{2} r^{2}-r^{2} \sinh \frac{1}{2} r^{2}}{\sinh ^{3} \frac{1}{2} r^{2}} .
\end{aligned}
$$

4.3. Pair correlation in higher dimensions. The limit pair correlation measure is given by

$$
\begin{aligned}
\widetilde{K}_{2}^{\infty}(z, w) & =\lim _{N \rightarrow \infty} N^{2(m-1)} \widetilde{K}_{2}^{N}\left(\frac{z}{\sqrt{N}}, \frac{w}{\sqrt{N}}\right) \\
& =\vec{K}_{2}^{\infty}(z, w) \wedge \frac{1}{(m-1) !}\left(\frac{i}{2} \partial \bar{\partial}|z|^{2}\right)^{m-1} \wedge \frac{1}{(m-1) !}\left(\frac{i}{2} \partial \bar{\partial}|w|^{2}\right)^{m-1}
\end{aligned}
$$


(The scaling $N^{2(m-1)}$ comes from the fact that $N \omega\left(\frac{z}{\sqrt{N}}\right)=N\left(\tau_{\sqrt{N}}\right)_{*} \omega \rightarrow \frac{i}{2} \partial \bar{\partial}|z|^{2}$.) We now compute $\widetilde{K}_{2}^{\infty}$ for the case of a manifold of general dimension $m>1$. It is convenient to express this measure in terms of the expected density of zeros

$$
\widetilde{K}_{1}^{\infty}(z)=\lim _{N \rightarrow \infty} N^{m-1} \widetilde{K}_{1}^{N}\left(\frac{z}{\sqrt{N}}\right)=\frac{m}{\pi} d V_{\mathbb{C}^{m}}=\frac{1}{\pi(m-1) !}\left(\frac{i}{2} \partial \bar{\partial}|z|^{2}\right)^{m} .
$$

We have the following explicit universal formula for the limit pair correlation measure. In particular, it gives the scaling limit pair correlation for the zeros of SU $(m+1)$-polynomials.

Theorem 4.2. Suppose $\operatorname{dim} M=m>1$. Then

$$
\widetilde{K}_{2}^{\infty}(z, w)=\left[\gamma_{m}\left(\frac{1}{2}|z-w|^{2}\right)\right] \widetilde{K}_{1}^{\infty}(z) \wedge \widetilde{K}_{1}^{\infty}(w),
$$

where

$$
\begin{aligned}
\gamma_{m}(t)= & \frac{\left[\frac{1}{2}\left(m^{2}+m\right) \sinh ^{2} t+t^{2}\right] \cosh t-(m+1) t \sinh t}{m^{2} \sinh ^{3} t}+\frac{m-1}{2 m} \\
= & \frac{(m-1)}{2 m} t^{-1}+\frac{m-1}{2 m}+\frac{(m+2)(m+1)}{6 m^{2}} t \\
& \quad-\frac{(m+4)(m+3)}{90 m^{2}} t^{3}+\frac{(m+6)(m+5)}{945 m^{2}} t^{5}+O\left(t^{7}\right) .
\end{aligned}
$$

Proof. By (36) and (50), again writing $\zeta=z-w$ (except this time $\zeta \in \mathbb{C}^{m}$ ),

$$
\begin{aligned}
\widetilde{K}_{2}^{\infty}(z, w) & =\left[1+\frac{4}{m^{2}} \sum_{j, k=1}^{m} \frac{\partial^{2}}{\partial z_{j} \partial \bar{z}_{j}} \frac{\partial^{2}}{\partial w_{k} \partial \bar{w}_{k}} G\left(e^{-\frac{1}{2}|z-w|^{2}}\right)\right] \widetilde{K}_{1}^{\infty}(z) \wedge \widetilde{K}_{1}^{\infty}(w) \\
= & {\left[1+\frac{1}{4 m^{2}} \Delta_{\zeta}^{2} G\left(e^{-\frac{1}{2}|\zeta|^{2}}\right)\right] \widetilde{K}_{1}^{\infty}(z) \wedge \widetilde{K}_{1}^{\infty}(w) } \\
= & {\left[1+\frac{1}{4 m^{2}}\left(\frac{d^{2}}{d r^{2}}+\frac{2 m-1}{r} \frac{d}{d r}\right)\left(m \log \left(1-e^{-r^{2}}\right)+\frac{r^{2}}{e^{r^{2}}-1}\right)\right] \widetilde{K}_{1}^{\infty}(z) \wedge \widetilde{K}_{1}^{\infty}(w) . }
\end{aligned}
$$

Computing the Laplacian in (52) leads to the stated formula.

Note that if we substitute $m=1$ in the expression for $\gamma_{m}(t)$, we obtain Hannay's function $H(t)$. However for the case $m>1$, the limit measure is absolutely continuous on $\mathbb{C}^{m} \times \mathbb{C}^{m}$, whereas in the one-dimensional case, there is a self-correlation delta measure.

\section{REFERENCES}

[BD] P. Bleher and X. Di, Correlations between zeros of a random polynomial, J. Stat. Phys. 88 (1997), 269-305.

[BSZ] P. Bleher, B. Shiffman and S.Zelditch, Universality and scaling of correlations between zeros on complex manifolds (preprint 1999).

[BBL] E. Bogomolny, O. Bohigas, and P. Leboeuf, Quantum chaotic dynamics and random polynomials, J. Stat. Phys. 85 (1996), 639-679.

[GH] P. Griffiths and J. Harris, Principles of Algebraic Geometry, Wiley-Interscience, N.Y. (1978).

[Ha] J. H. Hannay, Chaotic analytic zero points: exact statistics for those of a random spin state, $J$. Phys. A: Math. Gen. 29 (1996), 101-105.

[SZ] B. Shiffman and S. Zelditch, Distribution of zeros of random and quantum chaotic sections of positive line bundles, Commun. Math. Phys. 200 (1999), 661-683. 
[Ti] G. Tian, On a set of polarized Kähler metrics on algebraic manifolds, J. Diff. Geometry 32 (1990), 99-130.

[Ze] S. Zelditch, Szegö kernels and a theorem of Tian, Int. Math. Res. Notices 6 (1998), 317-331.

Department of Mathematical Sciences, IUPUi, Indianapolis, IN 46202, USA

E-mail address: bleher@math.iupui.edu

Department of Mathematics, Johns Hopkins University, Baltimore, MD 21218, USA

E-mail address: shiffman@math.jhu.edu

Department of Mathematics, Johns Hopkins University, Baltimore, MD 21218, USA

E-mail address: zel@math.jhu.edu 